\title{
Schmidt, Vivien A. (2020): Europe's Crisis of Legitimacy. Governing by Rules and Ruling by Numbers in the Eurozone
}

\author{
Oxford: Oxford University Press. 384 Seiten. $£ 26,49$
}

\author{
Stefan Wallaschek
}

Angenommen: 23. September 2021 / Online publiziert: 13. Oktober 2021

(C) Der/die Autor(en) 2021

In „Europe's Crisis of Legitimacy“ legt Vivien Schmidt ihre langjährige Forschung zur Eurokrise dar und nutzt dabei ihre hervorragenden Kenntnisse der EU durch ihr vielfaches „Insider-Wissen“ und Nähe zu den europäischen Institutionen und verbindet in dem Werk ihre drei zentralen Forschungsfelder: Fragen der Demokratie in Europa und der Europäischen Union, veränderte Formen demokratischer Legitimation und ihre Arbeiten zum diskursiven Institutionalismus.

Im ersten Teil setzt sich Schmidt ausführlich mit verschiedenen Typen von Legitimität (Input, Output, Throughput) auseinander und wie diese im Multi-LevelGovernance-System der EU verortet werden können. Schmidt argumentiert, dass sich die Input-Legitimität vor allem auf die nationale Ebene stützt, während die Output- und Throughput-Legitimität sich vor allem auf der EU-Ebene konzentrieren. Dies führe zu einer problematischen „split-level legitimacy“ (S. 56), die sich besonders in der Eurokrise verdeutlicht hat. Im zweiten Teil des Buches fokussiert die Autorin auf die Throughput-Legitimität anhand von fünf Kriterien (,efficacy“, ,accountability“, „,transparency“, ,inclusiveness“ und „openness“) und analysiert die Diskurse und Handlungsweisen der zentralen EU-Institutionen (Europäischer Rat, Europäische Zentralbank, Europäische Kommission und Europäisches Parlament) in der Eurokrise. Schmidt zeigt gekonnt auf, wie die Eurokrise anfangs ein Stück weit selbst durch das zögerliche Handeln einiger EU-Mitgliedsstaaten, allen voran Deutschland, befeuert wurde und damit eine Krisendynamik auslöste, die kaum noch zu kontrollieren war. Dabei hätte das Mandat der Europäischen Zentralbank (EZB) als ,Lender of Last Ressort“ frühzeitig entsprechend ausgelegt werden können, um die Krise einzudämmen. Als Krisenreaktion wurde ein Mix aus ordound neoliberalen Ideen („Brussels-Frankfurt consensus“, S. 109) durchgesetzt und

Stefan Wallaschek $(\triangle)$

Europa-Universität Flensburg, Flensburg, Deutschland

E-Mail: stefan.wallaschek@uni-flensburg.de 
schließlich wurde dann umso strikter re(a)giert (von Schmidt bereits früher als ,governing by rules and ruling by numbers" bezeichnet). Nichtsdestoweniger zeigt die Autorin auch auf, dass die Krisenpolitik vielfach angepasst oder sich gewandelt hat und damit ein ,reinterpreting the rules by stealth“ (S. 112) vollzogen wurde. So z. B. vom technokratischen Politikverständnis des Kommissionspräsidenten Barroso zum politischen Kompromisspräsidenten Juncker, der mehr auf Ausgleich zwischen Kreditgeber- und Kreditnehmerstaaten bedacht war. Dabei hat Juncker jedoch kaum grundlegend die Politik geändert, sondern mehr Flexibilität und Interpretationsraum für Maßnahmen eröffnet. Schmidt zeigt also vielfach den Policy-Wandel und die Anpassung der Maßnahmen im Krisenverlauf an, wodurch sie ein differenziertes Bild der Eurokrisenmaßnahmen zeichnet. Nichtsdestoweniger demonstriert sie, dass der Wandel meist zu spät kam, erst gegen Widerstände durchgesetzt wurde und meist nicht weit genug reichte, um einen tatsächlichen Wechsel durchzusetzen und damit ein wirkliches Ende der Krise einzuleiten. Gerade indem Schmidt die Eurokrise und deren legitimatorischen Defizite in den einzelnen EU-Institutionen rekonstruiert, trägt sie zu einem besseren Verständnis ,,der“ EU in der Eurokrise bei und wie die Institutionen selbst miteinander im Konflikt um geeignete Krisenmaßnahmen standen. Es muss jedoch auch konsterniert werden, dass die Krisenursachen nicht behoben wurden, wodurch der nächste Ausbruch nicht allzu unwahrscheinlich ist.

Teil 3 fokussiert auf die Input- und Output-Legitimität in der Eurokrise. Schmidt zeigt auf, wie ineffektiv die Austeritätsmaßnahmen und das Eurokrisenmanagement durch EU und Mitgliedstaaten waren. Die bisherige Output-Legitimierung der EU, dass sie sich positiv auf das Leben der Menschen auswirke (Stichwort: Personenfreizügigkeit oder gemeinsamer Währungsraum), ist durch die Eurokrise und den Umgang mit der Krise substanziell geschwächt worden. Ebenso hat die Input-Legitimität gelitten, weil die EZB nicht demokratisch legitimiert wird, aber eine zentrale Rolle beim Krisenmanagement eingenommen hat. Die Etablierung der Troika/ Institutionen (EZB, Europäische Kommission und Internationaler Währungsfonds) sowie die zahlreichen (nächtlichen) Krisengipfel zwischen Europäischem Rat und Kommission bei gleichzeitig schwacher Beteiligung und Kompetenz des Europäischen Parlaments haben die Input-Legitimität der EU ebenfalls nicht verbessert. Da sie überdies noch das Erstarken euroskeptischer und populistischer Parteien anführt, die aus der Eurokrise (und Migrationskrise) vielfach gestärkt hervorgegangen sind, weil sie erfolgreich gegen ,die“ EU und für mehr nationale Souveränität mobilisieren (von Schmidt als ,national ,politics against policy““ bezeichnet, S. 259), demonstriert die Autorin eindrücklich, dass es um die Legitimität der EU nicht gut bestellt ist.

Es bleibt etwas offen, inwiefern die Analyse der Throughput-Legitimität besser erklärt, wie die Eurokrise verlaufen ist und wie sich das Bild der Eurokrise durch diese Analyse verändert. Zum anderen frage ich mich, warum der dritte Teil nicht, wie schon Schmidt mal geplant (S. 20-21), nach vorne gezogen wurde. Viele Verweise auf den Verlauf der Krise und die Auswirkungen auf Krisenstaaten wie Griechenland oder Irland sind wichtige Kontextfaktoren, die für die Erklärung der ThroughputLegitimität zentral sind. Schmidt verzahnt Teil 2 und 3 gekonnt, aber nach Teil 3 bleibt man recht überzeugt zurück, dass es vor allem die Output-Legitimation war, die hier gelitten hat und die Throughput-Legitimität hingegen weniger relevant war. 
Das Fazit schlägt dann auch nur recht knapp den Bogen zurück zur ThroughputLegitimität. Hier wäre es womöglich strategisch besser und argumentativ überzeugender gewesen, wenn die Autorin erst Output- und Input-Fragen diskutiert hätte, um dann im zentralen und auch umfangreichsten Teil aufzuzeigen, was die Analyse der Throughput-Dimension leisten kann und was bisher unbeachtet geblieben ist.

Als Konsequenz aus den legitimatorischen Defiziten auf allen Ebenen plädiert Schmidt für ein stärker differenziertes Europa oder wie sie schreibt: ,The only Eurozone problem capable of responding to all such problems is one that provides for greater differentiation and decentralization in Eurozone governance" (S. 294). Auch wenn diese die Fliehkräfte der EU verringern und mehr nationale Mitsprache durch die Parlamente einbringen könnte, bleibt fraglich, inwiefern diese weitere Ausdifferenzierung zu einem besseren Verständnis der EU in der Bevölkerung beiträgt. Schaffen die z.B. von Schmidt vorgeschlagenen länderspezifischen Schulden- und Defizitregeln durch die EZB (statt „One-size-fits-all“-Regeln) mehr Akzeptanz in der Bevölkerung? Damit steigt die Komplexität von Entscheidungsprozessen in der EU und erfordert mehr Wissen der Bürger*innen darüber, welche Mitgliedsstaaten in einem Politikfeld abstimmungsberechtigt sind und welche Konsequenzen sich daraus ergeben, um der Politik in der EU besser folgen zu können. Und ob z. B. im Fall einer Entscheidung über ein EU-Budget nach der Herkunft der Europaabgeordneten unterschieden (Eurozonenmitglied oder nicht) (S. 301) eine bessere Politikvermittlung schafft, bleibt ebenfalls fraglich. Inwiefern also ein ,soft-core Europe“ (S. 291) tatsächlich eine nachhaltige Zukunft Europas darstellen kann, ist weiter zu diskutieren und wird sich auch an den kommenden politischen Konstellationen in der EU entscheiden.

„Europe's Crisis of Legitimacy“ reiht sich in die umfangreiche Literatur zur Eurokrise ein und liefert besonders mit seinem Fokus auf die Legitimationsprozesse einen wichtigen Beitrag für die politikwissenschaftliche EU-Forschung. Wer die Arbeiten der Autorin in den letzten Jahren zum Krisenmanagement und zur Legitimationskrise der EU verfolgt hat, wird womöglich nicht so viele neue Erkenntnisse gewinnen. Die größte Stärke des Buchs ist demnach vielmehr die Form selbst und damit ein Plädoyer für das Schreiben einer Monografie. Es erlaubt der Autorin, eingehender Konzepte und theoretische Debatten zu diskutieren sowie die Prozesse und Entscheidungen in der EU detailliert zu rekonstruieren. Beispielsweise ist es in einem Zeitschriftenartikel schlicht nicht möglich, die Throughput-Legitimitation in den vier EU-Institutionen vergleichend zu analysieren (S. 117-222). Es ist also auch das Buchformat und dessen Vorzüge, sich umfassend und detailliert mit dem Forschungsgegenstand auseinanderzusetzen, die das Buch lesens- und empfehlenswert machen.

Funding Open Access funding enabled and organized by Projekt DEAL.

Open Access Dieser Artikel wird unter der Creative Commons Namensnennung 4.0 International Lizenz veröffentlicht, welche die Nutzung, Vervielfältigung, Bearbeitung, Verbreitung und Wiedergabe in jeglichem Medium und Format erlaubt, sofern Sie den/die ursprünglichen Autor(en) und die Quelle ordnungsgemäß nennen, einen Link zur Creative Commons Lizenz beifügen und angeben, ob Änderungen vorgenommen wurden. 
Die in diesem Artikel enthaltenen Bilder und sonstiges Drittmaterial unterliegen ebenfalls der genannten Creative Commons Lizenz, sofern sich aus der Abbildungslegende nichts anderes ergibt. Sofern das betreffende Material nicht unter der genannten Creative Commons Lizenz steht und die betreffende Handlung nicht nach gesetzlichen Vorschriften erlaubt ist, ist für die oben aufgeführten Weiterverwendungen des Materials die Einwilligung des jeweiligen Rechteinhabers einzuholen.

Weitere Details zur Lizenz entnehmen Sie bitte der Lizenzinformation auf http://creativecommons.org/ licenses/by/4.0/deed.de. 